\title{
A self-management mechanism to manage non- cooperative behaviors in LGDM-based supply chain risk mitigation
}

\author{
Sihai Zhao, Yucheng Dong \\ Business School \\ Sichuan University \\ Chengdu, China \\ shzhao@stu.scu.edu.cn \\ ycdong@scu.edu.cn
}

\author{
Hengjie Zhang \\ Business School \\ Hohai University \\ Nanjing, China \\ hengjiezhang@hhu.edu.cn
}

\author{
Francisco Chiclana \\ Centre for Computational \\ Intelligence \\ De Montfort University \\ Country Leicester, UK \\ chiclana@dmu.ac.uk
}

\author{
Enrique Herrera-Viedma \\ Department of Computer Science \\ and Artificial Intelligence \\ University of Granada \\ Country Granada, Spain \\ viedma@decsai.ugr.es
}

\begin{abstract}
Large-scale group decision making (LGDM) is becoming more and more common, and how to assure the security and quality of the decision making process has become a hot topic. Supply chain risk mitigation is a complex LGDM problem involving in many stakeholders. In the decision making process, a group of experts aims at reaching a consensus among alternatives in which non-cooperative behaviors often appear. Some experts might designedly form a small alliance and change their preferences in a direction against consensus with the aim to foster the alliance's own interests. In this study, we present a novel large-scale consensus reaching framework based on a selfmanagement mechanism to manage non-cooperative behaviors. In the proposed framework, experts are classified into different subgroups using a clustering method, and they provide their evaluation information, i.e., the multi-criteria mutual evaluation matrices (MCMEMs), regarding the obtained subgroups based on their performance. The subgroups' weights are generated dynamically from the MCMEMs, which are in turn used to update experts' weights. This mechanism allows penalizing the weights of the experts with non-cooperative behaviors. Detailed comparison analysis is presented to verify the validity of the proposed consensus framework for supply chain risk mitigation.
\end{abstract}

Keywords-consensus reaching process, large-scale group decision making, non-cooperative behaviors, supply chain risk mitigation, self-management mechanism

\section{INTRODUCTION}

Supply chain risk management $[20,31]$ is very important for a company, and there are many elements and stakeholders to be considered in this complex decision process. To mitigate the supply chain risk, a group of experts could be asked to make a choice among several alternatives, which can be regarded as a group decision making process.

Group decision making (GDM) [12] refers to a process in which a group of experts aim at obtaining a collective solution based on their opinions on a decision problem. And the consensus reaching process (CRP) $[3,6,8,17,23,24]$ is usually used to help improve the consensus level among experts.
In the past few years, with the development of information technology and society, decision contexts become more and more complex as a large number of stakeholders are often involved, as it is the case in supply chain risk management contexts. To cope with this type of decision situation, a number of large-scale group decision models have been proposed [18, $21,22,29,30]$. In a large-scale consensus reaching process (LCRP), because experts usually are from different domains and have different interests, they might adopt non-cooperative behaviors to achieve their own goals. For example, to solve a supply chain risk management problem a number of experts are going to make a choice among several alternatives. There may be situations that some experts will form an alliance and express their preferences in a direction against consensus to benefit. Thus, managing experts' non-cooperative behaviors is the key to assure the security and quality of the LCRP outcome. In the existing literature, two mainstream research approaches have been developed to deal with non-cooperative behaviors in the GDM: (1) managing non-cooperative behaviors in the aggregation process of the GDM $[25,26]$, which mainly analyzes the influence of the non-cooperative behaviors on the aggregation outcome; (2) managing noncooperative behaviors in the consensus process of the GDM [9, $18,21]$, which focuses on how to achieve a consensus under the presence of non-cooperative behaviors.

By analyzing existing non-cooperative behavior studies, we find that there are still some gaps should be filled: (1) models in $[25,26]$ only discuss the non-cooperative behaviors in the aggregation process in GDM problems. However, noncooperative behaviors often appear in the CRP, which presents more complex characteristics; $(2)$ models in $[18,21]$ to manage non-cooperative behaviors in the CRP depend on a moderator. However, in a practical decision problem, it could be difficult for a moderator to carry out such heavy and complicated task. (3) model in [9] manages non-cooperative behaviors based on mutual evaluation matrices in the CRP. However, in LCRP the mechanism cannot work well because it could be difficult to manage mutual evaluation matrices among a large group of experts. 
Motivated by the challenge to cope with non-cooperative behaviors in LCRP of supply chain risk management, in this study we propose a novel large-scale consensus framework based on a self-management mechanism, which allows penalizing the weights of the experts with non-cooperative behaviors.

The rest of this paper is organized as follows: Section II introduces the preliminaries. Section III proposes the novel consensus framework based on a self-management mechanism for supply chain risk mitigation. And then detailed simulation analysis are presented in section IV to verify the validity of the proposed framework. Finally, conclusion and future work are concluded in Section V.

\section{PRELIMINARIES}

This section introduces some basic knowledge regarding the additive preference relations, consensus reaching process and the LCRP.

\section{A. Additive preference relations}

There are two basic elements in a GDM problem: a set of alternatives, $X=\left\{x_{1}, x_{2}, \ldots, x_{n}\right\}(n \geq 2)$; and a set of experts, $E=\left\{e_{1}, e_{2}, \ldots, e_{m}\right\}(m \geq 2)$. Each expert can express his/her preference on $X$ using different preference representation structures $[4,7,11,15]$. Additive preference relation which is widely used in many GDM models is also adopted in this paper.

Definition 1 (Additive preference relation). An expert $e_{k}$ expresses his/her preferences on a set of alternatives $X$ by a matrix, $P^{k}=\left(p_{i j}^{k}\right)_{n \times n}$, in which $p_{i j}^{k} \in[0,1]$ denotes the expert's preference degree of alternative $x_{i}$ over $x_{j}$. It is assumed that the preference relation satisfies the additive reciprocity property, i.e., $p_{i j}+p_{j i}=1, \forall i, j$.

For simplicity, we call additive preference relations simply preference relations below.

\section{B. Consensus reaching process}

Many CRP models based on preference relations have been proposed in the specialized literature, which normally consist of a consensus process and a selection process.

(1) Consensus process aims at improving the consensus level among experts $[5,13,18]$, and it comprises two parts: consensus measure and feedback adjustment.

(a) Consensus measure $[5,13,18]$ is used to compute the level of agreement among experts. In this paper, we adopt the approach presented in [18] which is described below.

For a pair of experts $\left(e_{k}, e_{l}\right)$, a preference similarity matrix, $P S=\left(p s_{i j}^{k l}\right)_{n \times n}$, is defined, where $p s_{i j}^{k l}=1-\left|p_{i j}^{k}-p_{i j}^{l}\right|$. Let $\omega_{k}$ and $\omega_{l}$ be the weights of experts $\left(e_{k}, e_{l}\right)$, respectively. The following consensus matrix, $C M=\left(c m_{i j}\right)_{n \times n}$, with element

$$
c m_{i j}=\sum_{k=1}^{m-1} \sum_{l=k+1}^{m} \omega_{k l} p s_{i j}^{k l} / \sum_{k=1}^{m-1} \sum_{l=k+1}^{m} \omega_{k l}
$$

and $\omega_{k l}=\min \left\{\omega_{k}, \omega_{l}\right\}$ is computed [18]. The consensus level can be computed at the following three different levels:

(i) Consensus level on a pair of alternatives $\left(x_{i}, x_{j}\right)$,

$$
c p_{i j}=c m_{i j}
$$

(ii) Consensus level on alternative $x_{i}$,

$$
c a_{i}=\sum_{j=1, j \neq i}^{n} c m_{i j} /(n-1)
$$

(iii) Collective consensus level,

$$
c l=\sum_{i=1}^{n} c a_{i} / n
$$

(b) Feedback adjustment [9, 18] is used to help experts modify their preferences to improve the consensus level among experts. In this paper, this process is described below.

Let $P^{k}=\left(p_{i j}^{k}\right)_{n \times n}$ be the preference relation of expert $e_{k}$, $P^{c}=\left(p_{i j}^{c}\right)_{n \times n}$ be the collective preference relation, and $\overline{P^{k}}=\left(\overline{p_{i j}^{k}}\right)_{n \times n}$ be the adjusted preference relation of expert $e_{k}$. The adjustment process obeys following rules:

$$
\begin{cases}\overline{p_{i j}^{k}} \in\left[\min \left(p_{i j}^{k}, p_{i j}^{c}\right), \max \left(p_{i j}^{k}, p_{i j}^{c}\right)\right], & \text { if } i>j \\ \overline{p_{j i}^{k}}=1-\overline{p_{i j}^{k}}, & \text { if } i<j\end{cases}
$$

(2) Selection process aims to help experts find a solution to a decision problem. It is usually comprises two stages [9].

(a) Aggregation phase is used to obtain the collective preference relation, $p^{c}=\left(p_{i j}^{c}\right)_{n \times n}$. In this study, the WA operator is adopted in this process:

$$
p_{i j}^{c}=\omega_{1} p_{i j}^{1}+\omega_{2} p_{i j}^{2}+\ldots+\omega_{k} p_{i j}^{k}+\ldots+\omega_{m} p_{i j}^{m}=\sum_{k=1}^{m} \omega_{k} p_{i j}^{k}
$$

where $\omega_{k} \in[0,1]$ is the weight of expert $e_{k}$ and $\sum_{k=1}^{m} \omega_{k}=1$.

(b) Exploitation phase is used to rank the alternatives. This is done by associating a collective preference value, $C V_{i}$, to each alternative $x_{i}$. In this paper we use the following method to calculate $C V_{i}$.

$$
C V_{i}=\sum_{j=1}^{n} p_{i j}^{c} / n, i=1,2 \ldots, n
$$


According to Eq. (7), a ranking of alternatives can be obtained.

\section{Large-scale consensus reaching process}

Different from traditional GDM with a small number of experts participating in, the LCRP problem involves a large number of experts. Numerous methods have been proposed to cope with this type of problem in the existing literature, which usually obey the following scheme (e.g., [18]): firstly, a clustering method is adopted to classify the experts into a number of subgroups; then, a consensus process is utilized to improve the consensus level among experts; and finally, a selection method is used to obtain a collective group preference and the ranking of alternatives.

From above analysis, it is obvious that the most prominent feature of LCRP models is the application of a clustering process. In existing literature, there are many clustering methods available $[2,14,16]$ to perform this task. In this study the method proposed in [16] is adopted.

\section{THE LARGE-SCALE CONSENSUS FRAMEWORK BASED ON A SELF-MANAGEMENT MERCHANISM FOR SUPPLY CHAIN RISK MITIGATION}

This section proposes the LCRP problem in supply chain risk management with non-cooperative behaviors, and formally presents its resolution framework.

\section{A. LCRP problem in supply chain risk management with non-cooperative behaviors}

In a LCRP problem in supply chain risk management, some experts may collude with other experts and adopt noncooperative behaviors to further their own goals. As mentioned in Section I, the consensus building among a large number of experts has become a hot topic of research, and noncooperative behaviors usually appear in LCRP. Therefore it is important to study it and to tackle it with appropriate theoretically based models, and it is necessary to design a LCRP framework to cope with this situation.

\section{B. The large-scale consensus framework for supply chain risk mitigation}

As mentioned in section II-C, the resolution of LCRP problems usually obeys a scheme consisting of three different parts: clustering process, consensus process and selection process [18]. By integrating experts' weights generated dynamically into the LCRP, we propose a novel large-scale consensus framework based on a self-management mechanism for supply chain risk mitigation. In the proposed framework, the following four parts are differentiated as Fig. 1.

(1) Clustering process. A clustering algorithm is applied to classify experts into a number of subgroups.

(2) Dynamically generating weights process. Experts provide the MCMEMs regarding subgroups, and then subgroups' weights can be obtained from the MCMEMs. Finally, experts' weights can be updated dynamically by the obtained subgroups' weights, which are presented in Section III-C.

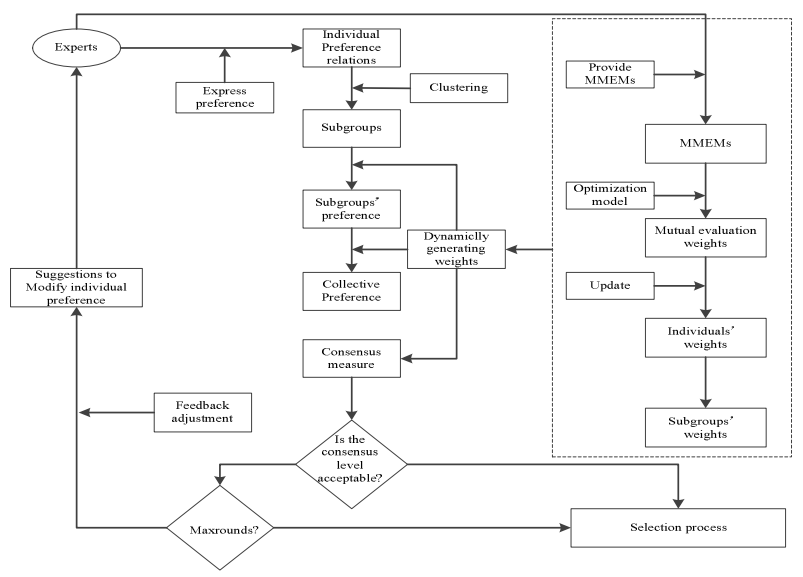

Fig. 1. The large-scale consensus framework for supply chain risk management

(3) Consensus process. Consensus measure is used to compute the consensus level among experts, and feedback adjustment is applied to help them improve the consensus level when the consensus level is below a satisfactory threshold .

(4) Selection process. Aggregation phase and exploitation phases are carried out to obtain the collective group preference relation and ranking of alternatives, respectively.

\section{Dynamically generating weights process}

Experts provide and update their MCMEMs regarding subgroups based on some criteria. And then subgroups' weights can be obtained from the MCMEMs. Finally, they are employed to update experts' weights.

(1) MCMEMs. It is assumed that there are $h$ subgroups $G=\left\{G_{1}, G_{2}, \ldots, G_{h}\right\}$ as a result of the application of the clustering process. Let $c=\left\{c_{1}, c_{2}, \ldots, c_{y}\right\}$ be a set of criteria of MCMEMs, and let $\kappa=\left(\kappa_{1}, \kappa_{2}, \ldots, \kappa_{y}\right)^{T}$ be a weight vector of criteria, in which $\kappa_{i} \in[0,1]$ and $\sum_{j=1}^{y} \kappa_{j}=1$. Experts will provide and update their MCMEMs about these subgroups in each consensus round.

Expert $e_{k}$ provides his/her MCMEMs, $V^{k}=\left(v_{i j}^{k}\right)_{h \times y}$, in which $v_{i j}^{k}$ denotes the evaluation value that expert $e_{k}$ assigns to the subgroup $G_{i}$ with respect to the criterion $c_{j}$. If $e_{k} \in G_{i}$, $v_{i j}^{k}=n u l l ;$ otherwise, $v_{i j}^{k} \in[0,100]$. The MCMEM of subgroup $G_{t}(t=1,2, \ldots, h), V^{G_{t}}=\left(v_{i j}^{G_{t}}\right)_{h \times y}$ is obtained by averaging the provided MCMEMs of the subgroup members:

$$
v_{i j}^{G_{t}}=\sum_{e_{k} \in G_{t}} v_{i j}^{k} /\left\|G_{t}\right\|
$$

where $\left\|G_{t}\right\|$ denotes the number of experts in this subgroup. And the MCMEM of the large group, $V^{G}=\left(v_{i j}^{G}\right)_{h \times y}$, is obtained by averaging all subgroups' MCMEMs. 


$$
v_{i j}^{G}=\sum_{t=1}^{h} v_{i j}^{G_{t}} /(h-1)
$$

(2) Obtaining the individuals' weights. $V^{G}$ is normalized $\overline{V^{G}}$ as follows.

$$
\left\{\begin{array}{l}
\overline{v_{i j}^{G}}=v_{i j}^{G} / \sum_{i=1}^{h} v_{i j}^{G}, \text { for the benefit criterion } a_{j}, j=1,2, \ldots, y \\
\overline{v_{i j}^{G}}=\frac{1 / v_{i j}^{G}}{\sum_{i=1}^{h}\left(1 / v_{i j}^{G}\right)}, \text { for the } \cos t \text { criterion } a_{j}, j=1,2, \ldots, y
\end{array}\right.
$$

The evaluation value that the large group assigns to the subgroup $G_{i}$ can be computed as $\zeta_{i}=\sum_{j=1}^{y} \kappa_{j} \overline{v_{i j}^{G}}$. A large value of $\zeta_{i}$ indicates that the large group believes the subgroup $G_{i}$ is quite important. Let $\lambda=\left(\lambda^{G_{1}}, \lambda^{G_{2}}, \ldots, \lambda^{G_{h}}\right)^{T}$ be the weight vector of $G=\left\{G_{1}, G_{2}, \ldots, G_{h}\right\}$. We can obtain the weights of subgroups by solving the following nonlinear programming model:

$$
\begin{aligned}
& \min \sum_{i=1}^{h}\left(\zeta_{i}-\lambda^{G_{i}}\right)^{2} \\
& \text { s.t. }\left\{\begin{array}{l}
\sum_{i=1}^{h} \lambda^{G_{i}}=1 \\
\lambda^{G_{i}} \geq 0, i=(1,2, \ldots, h)
\end{array}\right.
\end{aligned}
$$

By solving model (11), we have

$$
\lambda^{G_{i}}=\sum_{j=1}^{y} \kappa_{j} \overline{v_{i j}^{G}}
$$

Then, the obtained subgroups' weights are used to update the experts' weights. The updated weight of expert $e_{k} \in G_{i}$, $\overline{\omega_{k}}$, can thus be defined as follows:

$$
\overline{\omega_{k}}=\rho \omega_{k}+(1-\rho) \lambda^{G_{i}}
$$

where $\rho$ is a parameter used to control the amount of change in updating the experts' weights.

Finally, experts' updated weights are normalized:

$$
\overline{\overline{\omega_{k}}}=\overline{\omega_{k}} / \sum_{i=1}^{m} \overline{\omega_{i}}
$$

\section{APPLiCATION OF THE PROPOSED LARGE-SCALE CONSENSUS FRAMEWORK FOR SUPPLY CHAIN RISK MITIGATION}

In this section, we introduces a common non-cooperative behavior in LCRP of supply chain risk management contexts, and we applies the proposed large-scale consensus framework to manage it. Detailed simulation analysis is presented to verify the validity of the proposed framework.

\section{A. Non-cooperative behaviors in LCRP of supply chain risk management contexts}

In LCRP of supply chain risk management contexts, it is naturally that some experts might form an alliance which may adopt non-cooperative behaviors to further their own interests. To cope with this decision situation, firstly we introduce a concept of subgroup successive similarity: for any two subgroups in two successive consensus rounds, if the two subgroups share a large number of experts, then the two subgroups are considered similar and can be regarded as same. The successive similarity degree can be described as follows:

Let $G_{i, z-1}$ be a subgroup in consensus round $z-1, G_{j, z}$ be a subgroup in consensus round $z$, then

$$
S_{G_{i, z-1}, G_{j, z}}=\left\|G_{i, z-1} \cap G_{j, z}\right\| /\left\|G_{i, z-1} \cup G_{j, z}\right\|
$$

is considered as the similarity degree between the two subgroups. Let $\alpha(\alpha \in[0,1])$ be a given threshold. If $S_{G_{i, z-1}, G_{j, z}}>\alpha$, the two subgroups are considered the same.

Based on the concept of subgroup successive similarity, we consider the following common large-scale group noncooperative behaviors I.

\section{Large-scale group non-cooperative behavior I}

In LCRP of supply chain risk management contexts, experts' opinions will reach a consensus if they modify their preference relations according to the advice of feedback adjustment. However, a subgroup's preference relation might diverge from the group to further its own interests, and the deviation between the subgroup's preference relation and the group's preference relation could be very large. In this study, we call this type of behavior large-scale group non-cooperative behavior I.

Let $P^{\left(G_{i, z}\right)}$ be the preference relation of subgroup $G_{i, z}$ and $P^{(c, z)}$ be the group's preference relation in consensus round $z$. The deviation between them can be computed as follows:

$$
N^{G_{i, z}}=\sum_{i=1}^{n} \sum_{j=1, j \neq i}^{n}\left|p_{i j}^{\left(G_{i, z}\right)}-p_{i j}^{(c, z)}\right| /\left(n^{2}-n\right)
$$

Let $\beta(\beta \in[0,1])$ be a given threshold. If $N^{G_{i, z}} \geq \beta$, then we say that the subgroup $G_{i, z}$ features large-scale group noncooperative behavior I.

\section{B. Simulation experiments}

In this section, we design a simulation experiment to verify the validity of the proposed framework for managing noncooperative behaviors in LCRP.

In the simulation experiment, we generate the initial experts' preference relations randomly. After the clustering process, experts provide their MCMEMs. The MCMEMs involve three criteria: professional skill, fairness and cooperation. In existing literature, a number of methods are 
proposed to set the criterion weights in multiple criteria decision making (e.g., [1, 27, 28]). For instance, In this paper we set that all criterion weights are equal to manage large-scale group non-cooperative behavior I. We design simulation experiment I based on the following natural hypotheses 1 .

Hypothesis 1: If a subgroup features large-scale group noncooperative behavior I, then experts in other subgroups will decrease the evaluation value of this subgroup regarding the criterion " cooperation".

Simulation experiment $\mathrm{I}$ is described as follows.

Step 1: Let $z=1$. Initialize experts' preference relations and weights. And we set that $\bar{m}$ experts, $\bar{G}$, have noncooperative behavior I.

Step 2: Clustering. Classify experts into a number of subgroups $\left\{G_{1, z}, G_{2, z}, \ldots, G_{h, z}\right\}$ by the clustering method.

Step 3: MCMEMs. If $z=1$, for $e_{k} \in G_{t}(t=1,2, \ldots, h)$, $v_{t j}^{(k, z)}=n u l l$; for $e_{k} \notin G_{t, z}$, generate $v_{t j}^{(k, z)}$ randomly from interval [80,100]. If $z \geq 2$, according to hypothesis 1, experts in other subgroups will decrease the evaluation values of the subgroup $\bar{G}$ regarding the criterion "cooperation". The process is described as follows:

$$
\begin{aligned}
& v_{i j}^{(k, z)}=\text { null, } \quad e_{k} \in G_{i, z} \\
& v_{i j}^{(k, z)} \in[80,100], \quad \quad e_{k} \notin G_{i, z} \& G_{i, z} \neq \bar{G} \\
& \left\{v_{i j}^{(k, z)} \in[80,100], \quad e_{k} \notin G_{i, z} \& G_{i, z}=\bar{G} \& j=1,2\right. \\
& v_{i j}^{(k, z)} \in[\max (80-100 \theta, 0), 100-100 \theta], e_{k} \notin G_{i, z} \& G_{i, z}=\bar{G} \& j=3
\end{aligned}
$$

Step 4: Obtain experts' weights and collective preference relation. Based on obtained MCMEMs, use (8)-(12) to obtain subgroups' weights, $\lambda=\left(\lambda^{G_{1, z}}, \lambda^{G_{2, z}}, \ldots, \lambda^{G_{h, z}}\right)$. Then use the obtained subgroups weights to update experts' initial weights by (13)-(14) and obtain collective preference relation by (6).

Step 5: Consensus measure. Use (1)-(4) to obtain the consensus level $c l$ among experts. If $c l \geq \overline{c l}$ or $z \geq z_{\max }$, then go to step 7; otherwise, continue with the next step.

Step 6: Feedback adjustment. For $e_{k} \notin \bar{G}$, use (5) to modify their preference relations; for $e_{k} \in \bar{G}$, generate experts' preference relations, and guarantee that the deviation between the subgroup $\bar{G}$ and the large group is larger than $\beta$.

Let $z=z+1$, then go to step 2 .

Step 7: Output. If $c l \geq \overline{c l}$, then $s=1$; otherwise, $s=0$. Output $z$ and $s$.

Note 1: (1) the parameter $z$ denotes the iteration number to achieve a consensus among experts; (2) the parameter $s$ reflects whether the given consensus level $\overline{c l}$ can be achieved; (3) the parameter $\theta(\theta \in[0,1])$ denotes the penalty coefficient, with a larger $\theta$ value denoting a larger penalty strength.

\section{Comparitive analysis}

In this section, we compare the proposed large-scale group non-cooperative behaviors management framework against the traditional LCRP to verify the validity of our framework.

In traditional CRP models, experts' weights remain unchanged. The traditional methods can be also exported to manage large-scale GDM after some minor modifications. In our simulation experiment I, when deleting the dynamically generating weights process, we could obtain the traditional LCRP, i.e., we remove steps 3-4 in Simulation experiments I to obtain simulation experiment $\mathrm{I}^{*}$, which can be used to describe traditional LCRP.

Let $n=6, z_{\max }=5, \overline{c l}=0.9, \theta=0.2$, and $\bar{m}=10$. When setting different input parameters $m$ and $\beta$ for simulation methods I* and I, we run these two simulation experiments 1000 times, and we can obtaining the average values of $s$ and $z$. The simulation results are described in Fig. 2.
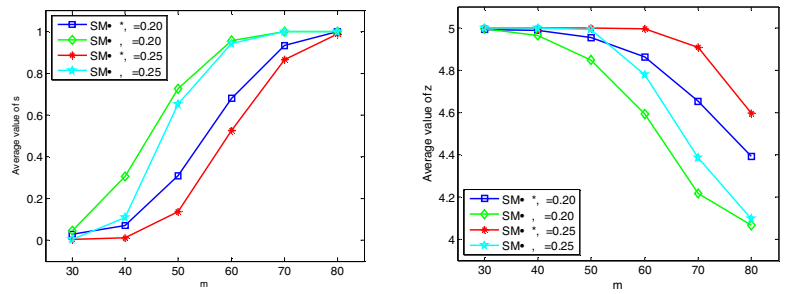

Fig. 2. Average $s$ and $z$ values in Simulation experiments I and $\mathrm{I}^{*}$ under different parameters $m$ and $\beta$

From Fig. 2, we have the following observations:

(1) The consensus success ratios in the proposed framework are clearly higher than those in the traditional LCRP, which means that the proposed framework can improve the success ratio of achieving a consensus among experts by managing the large-scale group non-cooperative behavior I.

(2) There are obviously fewer average consensus rounds in the proposed framework than those in the traditional LCRP, which means that the proposed framework can accelerate the speed to achieve a consensus among experts.

\section{CONCLUSION}

In LCRP of a practical supply chain risk management problem, it is common that some experts may collude with other experts and adopt non-cooperative behaviors to further their own goals. In this paper we propose a novel large-scale consensus framework based on a self-management mechanism for managing non-cooperative behaviors in this decision situation. In the proposed framework, experts will provide and update their MCMEMs based on subgroups' performance from which subgroups' weights could be obtained. Then experts' weights can be updated dynamically by the obtained subgroups' weights, and they are integrated into the LCRP. We consider a common large-scale group non-cooperative behavior in LCRP. If a subgroup is detected to use this noncooperative behavior, experts in other subgroups will decrease 
the evaluation values of this subgroup which allows decreasing the weights of experts in this subgroup. Also, we design detailed simulation experiment to verify the validity of the proposed framework.

In fact, social relationships $[8,10]$ among experts may also play a key role in the consensus building. Meanwhile, decision elements could be changed dynamically during the LCRP, examples of which are the experts' participation rate, or the number of available alternatives [19]. We believe that it could be very interesting in future work to investigate and cope with the social relations issues and dynamic elements under the selfmanagement mechanism-based framework in the large-scale group consensus building.

\section{REFERENCES}

[1] F.H. Barron, B.E. Barrett, "Decision quality using ranked attribute weights," Management Science, vol. 42, pp. 1515-1523, 1996.

[2] A. Baraldi, P. Blonda, "A survey of fuzzy clustering algorithms for pattern recognition," IEEE Transactions on Systems, Man, and Cybernetics Part B: Cybernetics, vol. 29, pp. 778-785, 1999.

[3] D. Ben-Arieh, T. Easton, B. Evans, "Minimum cost consensus with quadratic cost functions," IEEE Transactions on Systems, Man, and Cybernetics Part A: Systems and Humans, vol. 39, pp. 210-217, 2008.

[4] X. Chen, H.J. Zhang, Y.C. Dong, "The fusion process with heterogeneous preference structures in group decision making: A survey," Information Fusion, vol. 24, pp. 72-83, 2015.

[5] F. Chiclana, J.M. Tapia García, M.J. del Moral, E. Herrera-Videdma, "A statistical comparative study of different similarity measures of consensus in group decision making," Information Sciences, vol. 221, pp. 110-123, 2013.

[6] Y.C. Dong, H.J. Zhang, E. Herrera-Viedma, "Consensus reaching model in the complex and dynamic MAGDM problem," Knowledge-based Systems, vol. 106, pp. 206-219, 2016.

[7] Y.C. Dong, C.C. Li, Y.F. Xu, X. Gu, "Consensus-based group decision making under multi-granular unbalanced 2-tuple linguistic preference relations," Group Decision and Negotiation, vol. 24, pp. 217-242, 2015.

[8] Y.C. Dong, Z. G. Ding, L. Martínez, F. Herrera, "Managing consensus based on leadership in opinion dynamics," Information Sciences, vol. 397-398, pp. 187-205, 2017.

[9] Y.C. Dong, H.J. Zhang, E. Herrera-Viedma, "Integrating experts' weights generated dynamically into the consensus reaching process and its application in managing non-cooperative behaviors," Decision Support Systems, vol. 84, pp. 1-15, 2016.

[10] Y.C. Dong, M. Zhan, G. Kou, Z.G. Ding, H.M. Liang, "A survey on the fusion process in opinion dynamics," Information Fusion, vol. 43, pp. $57-65,2018$

[11] E. Herrera-Viedma, F. Herrera, F. Chiclana, "A consensus model for multiperson decision making with different preference structures," IEEE Transactions on Systems, Man and Cybernetics Part A: Systems and Humans, vol. 32, pp. 394-402, 2002.

[12] J. Kacprzyk, "Group decision making with a fuzzy linguistic majority," Fuzzy Sets and Systems, vol. 18, pp. 105-118, 1986.
[13] J. Kacprzyk, M. Fedrizzi, "A 'soft' measure of consensus in the setting of partial (fuzzy) preferences," European Journal of Operational Research, vol. 34, pp. 316-325, 1988.

[14] G. Klir, B. Yuan, Fuzzy Sets and Fuzzy Logic: Theory and Applications, Englewood Cliffs, 1995.

[15] W.Q. Liu, Y.C. Dong, F. Chiclana, F.J. Cabrerizo, E. Herrera-Viedma, "Group decision-making based on heterogeneous preference relations with self-confidence," Fuzzy Optimization and Decision Making, vol. 16:4, pp. 429-447, 2017.

[16] S.F. Liu, Y.G. Dang, Z.G. Fang, et al., Grey system theory and application, Beijing: Science Press, 2010.

[17] I. Palomares, L. Martínez, "A semisupervised multiagent system model to support consensus-reaching processes," IEEE Transactions on Fuzzy Systems, vol. 22, pp. 762-777, 2014

[18] I. Palomares, L. Martínez, F. Herrera, "A consensus model to detect and manage noncooperative behaviors in large-scale group decision making," IEEE Transactions on Fuzzy Systems, vol. 22, pp. 516-530, 2014.

[19] I.J. Pérez, F.J. Cabrerizo, E. Herrera-Viedma, "A mobile decision support system for dynamic group decision-making problems," IEEE Transactions on Systems, Man, and Cybernetics Part A: Systems and Humans, vol. 40, pp. 1244-1256, 2010.

[20] P. Finch, "Supply chain risk management," Supply Chain Management: An International Journal, vol. 9(2), pp. 183-196, 2004.

[21] F.J. Quesada, I. Palomares, L.Martínez, "Managing experts behaviors in large-scale consensus reaching process with uninorm aggregation operators," Applied Soft Computing, vol. 35, pp. 873-887, 2015.

[22] T. Wu, X.W. Liu, "An interval type-2 clustering solution for large-scale multiple-criteria group decision making problems," Knowledge-Based Systems, vol. 114, pp. 118-127, 2016.

[23] Z.B. Wu, J.P. Xu, "A consistency and consensus based decision support model for group decision making with multiplicative preference relations," Decision Support Systems, vol. 52, pp. 757-767, 2012.

[24] Z.B. Wu, J.P. Xu, "Managing consistency and consensus in group decision making with hesitant fuzzy linguistic," Omega, vol. 65, pp. 2840, 2016.

[25] R.R. Yager, "Penalizing strategic preference manipulation in multiagent decision making," IEEE Transactions on Fuzzy Systems, vol. 9, pp. 393-403, 2001

[26] R.R. Yager, "Defending against strategic manipulation in uninormbased multi-agent decision making," European Journal of Operational Research, vol. 141, pp. 217-232, 2002.

[27] K. Yoon, C.L. Hwang, Multiple attribute decision making: methods and applications, Springer, Berlin, 1981.

[28] H.J. Zhang, Y.C. Dong, X. Chen, "The 2-Rank consensus reaching model in the multigranular linguistic multiple-attribute group decisionmaking," IEEE Transactions on Systems, Man, and Cybernetics, in press.

[29] H.J. Zhang, Y.C. Dong, E. Herrera-Viedma, "Consensus building for the heterogeneous large-scale GDM with the individual concerns and satisfactions," IEEE Transactions on Fuzzy Systems, in press.

[30] Z. Zhang, C. Guo, L. Martínez, "Managing multi-granular linguistic distribution assessments in large-scale multi-attribute group decision making," IEEE Transactions on Systems, Man, and Cybernetics: Systems, vol. 47, pp. 3063 - 3076, 2017.

[31] G. A. Zsidisin, M. E. Smith, "Managing supply risk with eraly supplier involvement: a case study and recearch propositions," Journal of Supply Chain Management, vol.41(4), pp. 44-57, 2005. 